\title{
Prognostic impact of perioperative sputum colonization on early outcome after lung transplant
}

\author{
Taehwa Kim ${ }^{1}$, Hye Ju Yeo ${ }^{1}$, Do Hyung Kim², Jin Ho Jang ${ }^{1}$, Eunjeong Son ${ }^{1}$, Jin ook Jang ${ }^{1}$, Yun Seong Kim ${ }^{1}$ Woo Hyun Cho \\ ${ }^{1}$ Division of Pulmonary, Department of Internal Medicine, Pusan National University Yangsan Hospital, Yangsan, Korea \\ ${ }^{2}$ Department of Thoracic and Cardiovascular Surgery, Pusan National University Yangsan Hospital, Yangsan, Korea
}

Background: The case number of lung transplant is globally growing very fast. One of trouble some issue is acquired infection after lung transplant. The respiratory multi-drug resistant bacteria is a relative contra-indication for lung transplant. In the study, we aimed to assess the association between perioperative sputum colonization and clinical outcome after lung transplant.

Methods: The primary outcome was defined as the 1-year survival rate and secondary outcomes were early posttransplantation pneumonia (PTP), ventilator free day within 28 days, postoperation intensive care unit (ICU) stay and postoperative hospital of stay.

Results: A total of 76 patients underwent lung transplantation surgery from May 2013 to December 2019 in Pusan National University Yangsan Hospital. Of 76 patients, 52 patients were alive in 1-year survivor and 24 patients were not survivor. Clinical outcome had significant difference, especially early PTP and postoperative ICU stay (early PTP, P=0.009; postoperative ICU stay, $P=0.001)$. The early posttransplant pneumonia was diagnosed 37 . In the clinical outcomes according to early PTP, postoperative ICU stay, postoperative HOS and 1-year survival showed a large difference and had significant P-values (27.6 \pm 20.6 vs. 14.1 \pm 11.0 , $P=0.001 ; 76.0 \pm 50.9$ vs. $50.9 \pm 31.7, P=0.011 ; 20$ [54.1\%] vs. 32 [82.1\%], $P=0.009)$. The 1 -year survival rate was higher in without PTP group. The Kaplan-Meier survival curve showed patients with PTP had significantly higher 1-year mortality than without group $\left(\mathrm{X}^{2}=\right.$ 6.849, $P=0.009$ ).

Conclusions: Thirty-seven patients (48.7\%) were developed PTP in our medical center after lung transplantation surgery. If perioperative sputum colonization of recipients was positive, PTP was more occurred well rather than donors. Sputum colonization of recipient was more important factor than donor's. In the group with PTP, the proportion of multi-drug resistant was high than not PTP group. PTP was significantly associated with 1yr mortality. Therefore, this study suggests if perioperative sputum colonization of recipients is MDR, it need to concentration more than other microorganism.

Corresponding author: Woo Hyun Cho

E-mail: popeyes0212@hanmail.net

\section{(c) The Korean Society for Transplantation}

This is an Open Access article distributed under the terms of the Creative Commons Attribution Non-Commercial License (http://creativecommons.org/licenses/by-nc/4.0/) which permits unrestricted non-commercial use, distribution, and reproduction in any medium, provided the original work is properly cited. 